\title{
Challenging diagnoses of toxocariasis: a report of two cases
}

\author{
Maria Luisa Gastaldello, ${ }^{1}$ Marcello Bigliocca, ${ }^{2}$ Mauro Campanini ${ }^{2}$ \\ ${ }^{1}$ Department of Emergency Medicine, Università del Piemonte Orientale, Novara; ${ }^{2}$ Department of Internal Medicine II, \\ A.O.U. Maggiore della Carità, Novara, Italy
}

\begin{abstract}
The present study illustrates two cases of Toxocara canis infection: a 77-year-old man presenting with bilateral uveitis, sepsis, and non-convulsive status epilepticus (patient A); and a 63-year-old woman with a history of treated breast cancer, presenting with sight loss and paralysis of the right eye, neurological symptoms, pulmonary embolism, pneumonia, and a post traumatic vertebral fracture (patient B). Both diagnoses were challenging because the acute comorbidities withdrew the attention from the actual cause of the disease, and in one case the steroid treatment possibly affected the laboratory indexes. The lack of response to antibiotics and the involvement of specific organs and districts suggested parasitic infection. Indeed, immunoblot assay revealed the presence of specific T. canis immunoglobulin $\mathrm{G}$ antibodies. Both patients improved with albendazole 800 $\mathrm{mg} /$ die $(400 \mathrm{mg} \times 2)$ for 28 days; the neurological state normalized, while sight and eye motility remained impaired. Overall, the findings from these two toxocariasis cases indicate that the occurrence of severe neurological symptoms associated with ocular involvement should generate suspicion of parasitic infection rather than leading to differential diagnosis of common presentations such as bacterial and viral infections or autoimmune diseases.
\end{abstract}

Correspondence: Maria Luisa Gastaldello, Department of Emergency Medicine, Università del Piemonte Orientale, via Costa 9, 28100 Novara, Italy.

E-mail: isabrooks91@gmail.com

Key words: Toxocara canis; toxocariasis; ocular larva migrans; visceral larva migrans; infective uveitis.

Acknowledgments: we would like to thank Dr. Flavio Bobbio for his help in critically revising the literature and the Director of the Emergency Medicine Residency Program, Dr. Gian Carlo Avanzi, for his support in activities related to clinical research and publication. Furthermore, we would like to thank Dr. Mario Pirisi, Director of the Residency Program at A.O.U. Maggiore della Carità and Università del Piemonte Orientale, Novara, Italy, for the educational and financial support. We also thank Dr. Marcello Arsura for proofreading the manuscript.

Contributions: the three authors conceived the study; MLG wrote and edited the paper; MLG and MB contributed to the discussion of the main aspects of the two cases; MC supervised and revised the work.

Conflict of interest: the authors declare no potential conflict of interest.

Received for publication: 15 June 2018.

Revision received: 26 November 2018.

Accepted for publication: 13 December 2018.

This work is licensed under a Creative Commons Attribution NonCommercial 4.0 License (CC BY-NC 4.0).

(C) Copyright M.L. Gastaldello et al., 2019

Licensee PAGEPress, Italy

Italian Journal of Medicine 2019; 13:59-63

doi:10.4081/itjm.2019.1056

\section{Introduction}

Toxocara canis is a parasitic roundworm. It is not normally a human parasite, but it can cause human infection, called toxocariasis, as a result of ingestion of larvae of the dog ascarid. Dogs are in fact the reservoir for this parasite. ${ }^{1}$ Larvae penetrate the gut wall and encyst in tissues, while adult worms develop in the small intestine, where eggs are shed in the stool. Humans can become infected through direct contact with dogs or by ingesting contaminated food or soil. Indeed, one of our patients (patient A) had eaten snail extract in an herbal remedy, while the other (patient B) was the owner of two dogs.

The incidence of $T$. canis in dogs may vary in different countries. ${ }^{2}$ In Italy, $T$. canis is one of the most frequently found parasites in both private and kenneled dogs, even though the few studies on the domestic reservoir have not provided conclusive evidence. One study on the dog population in Northern Italy found that $6.6 \%$ of fecal and fur samples were positive for the nematode, and only a smaller percentage of those carried non-viable eggs. ${ }^{3}$ Another study, conducted in a rural region, found $T$. canis in $33.6 \%$ of the dogs examined. ${ }^{4}$ However, none of these studies established a clear correlation between the prevalence of the parasite in dogs and the incidence of symptomatic human toxocariasis.

Human infection may be asymptomatic or may involve several organs with non-specific lesions. Classical clinical presentation consists of visceral larva migrans (VLM) and ocular larva migrans (OLM), which have also been reported to affect an individual 
simultaneously. Other manifestations, subsequent to tissue colonization by the cysts and development of eosinophilic granulomas, are fever, headache, behavioral disturbances, anorexia, abdominal pain, rash, hepatomegaly, nausea, vomiting, wheezing, and pulmonary infiltrates. ${ }^{5,6}$ In addition, dermatologic manifestations as chronic urticaria, chronic pruritus, different forms of eczema, hypodermic nodules, and vasculitis have been reported. ${ }^{7}$

A particular form of $T$. canis infection is covert toxocariasis, which is characterized by non-specific symptoms and signs such as fever, abdominal pain, anorexia, nausea, vomiting, hepatomegaly, cough, headache, lethargy, behavioral and/or sleep disturbances, skin symptoms, limb pains, and lymphadenitis associated with high titers of anti-T. canis antibodies, with or without eosinophilia. ${ }^{8}$ Intriguingly, covert toxocariasis has been only described in children. ${ }^{9}$

In this study, we describe two patients where the diagnosis of toxocariasis proved to be rather difficult due to concomitant comorbidities and consequent technical problems.

Both patients provided informed oral consent to be reported in this manuscript.

\section{Case Reports}

\section{Patient A}

Patient A was a 77-year-old male with a recent myocardial infarction and sepsis by S. epidermidis. He had been diagnosed with monoclonal gammopathy of undetermined significance, anxious-depressive syndrome, hypothyroidism, and atherosclerosis. He lived at home with his wife. His family history did not provide any relevant information.

Five months prior admission to our department, he had been admitted to another hospital for an inferior ST-elevation myocardial infarction. At the time, he had developed sepsis due to $S$. epidermidis, anemia, and $E$. coli urinary tract infection. Subsequently, the patient had been treated with antibiotics and had fully recovered.

He came to our observation as he had developed bilateral eye inflammation, which had been initially diagnosed as conjunctivitis, cough, and fever. The general practitioner prescribed ceftriaxone for 7 days, with no response. Subsequently, the patient had an episode of syncope at home during the night. He was taken to the hospital and referred to the Internal Medicine Department. Blood tests revealed neutrophil leukocytosis and hyponatremia. Almost immediately, the patient developed non-convulsive status epilepticus. For this reason, he was transferred to the Neurology Department where he underwent a brain computed tomographic (CT) scan that did not identify any lesion or ischemic areas. An ocular echo, performed in order to investigate the eye inflammation, revealed bilateral papilledema, retinal detachment in the right eye and bilateral uveitis. Suspecting a meningoencephalitis, an antibiotic therapy with amoxicillin and vancomycin was started. A lumbar puncture was initially avoided because the patient was taking oral anticoagulants and undergoing two antiplatelet therapies. Serology for cytomegalovirus, Toxoplasma, Chlamydia, Syphilis, and Borrelia resulted negative, as well as quantiFERON-TB.

Brain magnetic-resonance imaging (MRI) scan revealed diffuse lesions compatible with chronic vasculopathy. In the occipital-inferior-medial area and in the cerebellum, several similar lesions were interpreted as areas of cerebromalacia without pathological enhancement. The thickening of the walls of both eye bulbs was of uncertain interpretation. Even after days of antibiotic therapy, there was no clinical improvement. The neurological state remained constantly compromised with lethargy and poor response to verbal stimuli. After a new fever peak of up to $40^{\circ} \mathrm{C}$ and persistence of C-reactive protein at a concentration of $15.56 \mathrm{mg} / \mathrm{dL}$, the patient developed respiratory insufficiency with pulmonary consolidation.

The patient was then moved back to the Internal Medicine Department, where antibiotics were suspended and substituted. However, many blood cultures resulted negative.

After reviewing and discussing the possible causes of uveitis, it was decided to perform a Western blot (WB) analysis for T. canis, with a positive result (IgG immunoblotting). Thus, a therapy with albendazole $800 \mathrm{mg} /$ die ( $400 \mathrm{mg}$ twice a day) for 28 days was administered. In this case, later identified as OLM, prednisone was administered, as this addition is indicated with large consensus in the literature in cases of multiorgan impairment. The clinical conditions improved, and the patient fully recovered after seven days. An antiepileptic therapy with levetiracetam was maintained for the whole stay and at home to prevent new episodes of seizures. Ophthalmic reevaluation reported reduced vitreitis, but sight in the right eye could not be regained because of the retinal detachment. The patient was then discharged, and follow-up controls were carried out at 7 and 14 days after discharge. His conditions were stable, but he never regained sight or motility of the right eye. The left eye recovered with reduction of papilledema. $\mathrm{He}$ was compliant with the antiepileptic therapy and had no more seizures. The blood exams were normal in terms of blood count, electrolytes, renal function, and C-reactive protein.

\section{Patient B}

Patient B was a 63-year-old overweight woman on hormonotherapy with a history of breast cancer, which 
had been surgically treated. She lived at home and could autonomously manage her daily activities. She was the mother of two daughters, apparently healthy. She owned two dogs. Her family history did not provide any relevant information. She had never smoked tobacco and denied alcohol abuse. She had been diagnosed with hypertension at age 30 , and she had undergone mammary quadrantectomy and chemotherapy plus hormonotherapy (anastrazole) for breast cancer at the age of 59 .

Five months before she came to our observation, she had suffered from a hypertensive crisis with epistaxis and had been therefore admitted to a district hospital. During the hospital stay, both eyes had become swollen and red. Subsequently, only the right eye had progressively worsened with exophthalmos, ptosis, paralysis, and sight loss. Failing to define the diagnosis, after two months, she was transferred to the Ophthalmology Department. Thyroid-stimulating hormone (TSH) and TSH receptor antibodies were unremarkable. Suspecting retro orbital cellulitis, several antibiotics were administered without any effect. Thus, MRI scan of the head and face was performed, revealing non-specific ischemic/vasculitic brain lesions (inconclusive neurological evaluation) and infiltration of the eye and periorbital tissue. Biopsy of the eye resulted in non-diagnostic sample (fatty tissue). Another biopsy described in the periorbital tissue an inflammatory pseudotumor. Prednisolone was started without effect.

As her neurological state had worsened leading to acute confusion, the patient was transferred to the Neurology Department. Suspecting a meningoencephalitis, a lumbar puncture was performed but provided no evidence of infection, with normal cell count. The whole central nervous system was examined with brain and spine MRI: the brain lesions were unchanged, while it was diagnosed a T4 vertebral fracture. The patient reported she had accidentally fallen at home one month earlier. Protein electrophoresis revealed hypogammaglobulinemia. Serological tests for hepatitis B and C viruses, and human immunodeficiency virus were negative. No electroencephalogram signs of epilepsy or anomalies were found. CT scan of chest and abdomen revealed pulmonary embolism, deep venous thrombosis, and pneumonia, without evidence of cancer. Positronemission tomographic scan showed reactive accumulation in lung and right eye. She was therefore referred to the Internal Medicine Department to investigate the cause of the disease.

At the time of admission, the patient presented with neurological malfunctions with lethargy, disorientation, and mild cognitive impairment. She reported abdominal epigastric and limb pain. She was blind in one eye, which was red and swollen with a periorbital inflammation and paralysis of eye muscles. The patient could not walk due to the intense back pain caused by the vertebral fracture. There was no clinical evidence of skin, mouth or breast lesions, lymphadenomegaly or articular swelling. The temperature was almost at any time between $37-37.5^{\circ} \mathrm{C}$.

Blood tests revealed anemia, and absence of leukocytosis or eosinophilia; C-reactive protein was constantly elevated over $15 \mathrm{mg} / \mathrm{dL}$, whereas other parameters were unremarkable. Several sets of blood cultures were negative. Neoplastic markers, autoantibodies, serology for Toxoplasma, Chlamydia and Borrelia were negative. In the hypothesis of a paraneoplastic syndrome, endoscopic exams of upper and lower digestive tract were performed, but there was no bleeding or lesions.

Given the recent experience from patient A, a WB for $T$. canis was performed with positive result. Thus, a therapy with albendazole $800 \mathrm{mg} / \mathrm{die}$ (400 $\mathrm{mg}$ twice a day) for 28 days was administered. According to the literature, prednisone should have also been administered in case of OLM suspicion. However, since the neurological disturbances quickly responded to albendazole, and considering that the patient had already undergone steroid treatment for months with side effects (e.g. hypokalemia, insomnia, and osteoporosis), we decided not to add prednisone to the therapy.

The patient rapidly became more responsive and the neurological state recovered to normality. Her right eye slowly became less swollen and regained some motility and partial reactivity to light. The improvement of her conditions made it possible to repair the vertebral fracture with percutaneous vertebroplasty. The patient was then discharged and follow-up controls were programmed. She was seen at day 7, 14 and, 21 following discharge. Her conditions were stable, and she claimed she had felt well until then, apart from a mild cough with clear excretus. No more episodes of fever or abdominal or limbs pain were reported. She slowly started to walk again, with assistance from her relatives. Eye examination revealed regaining of the motility of the rectus lateralis, which was still paralized at the end of the hospital stay. The patient reported she had not regained sight in the right eye as she could only see lights and shadows. C-reactive protein was normal.

\section{Discussion}

This study reports two cases of $T$. canis infection whose diagnoses were particularly challenging due to the presence of a wide range of symptoms and comorbidities, where even the most typical manifestations of toxocariasis were misinterpreted as a result of complex clinical backgrounds. Indeed, we were unable to initially detect the presence of the 
parasite or eosinophilic granulomas in our patients for different reasons. In one case (patient A), we could not perform the lumbar puncture required to carry out lab tests because the patient was taking oral anticoagulants. On the other hand, the samples from patient B resulted negative for $T$. canis and showed no eosinophilia.

The absence of eosinophilia in patient $B$ could have been caused by a presentation of covert toxocariasis, or it may have been simply the consequence of the longterm corticosteroid treatment that had been administered to the patient in the Ophthalmology Department. It is also known from other studies that there is no peripheral blood eosinophilia in most cases of OLM, presumably because of the low larval load. ${ }^{10}$ Furthermore, cell count or indirect signs of infection cannot be regarded as clear signs of $T$. canis infection as these factors are affected by the vital activity and localization of the parasite.

In our two cases, the involvement of the central nervous system and the eyes alongside the inefficacy of several antibiotics led us to suspect parasitic infection, where $T$. canis seemed the most likely etiologic factor because of positive $\operatorname{IgG}$ results.

The most specific approach for serodiagnosis of either generalized forms of toxocariasis (i.e. VLM) or covert toxocariasis is reported to be the use of enzyme-linked immunosorbent assay (TES-ELISA: Toxocara ES Ag ELISA), after which any positive result should be tested by immunoblotting. ${ }^{11}$ Unfortunately, our local laboratory was not equipped with the Toxocara TES-ELISA kit, but WB was readily available; hence, WB was performed without any previous tests.

As far as the imaging findings are concerned, although in the literature there is some evidence attesting that brain MRI patterns may help to distinguish among neurotoxocarosis manifestations, ${ }^{12,13}$ our findings indicate the need for caution when interpreting MRI findings from toxocariasis patients. Indeed, it is highly unlikely that the MRI findings alone are sufficient to confirm a diagnosis of toxocariasis due to the heterogeneity of the manifestations that can selectively affect the central or peripheral nervous system with a wide range of lesions. In addition, MRI cannot fully distinguish between previous undiagnosed lesions, such as those caused by chronic hypertensive brain vasculopathies, and new ones.

A recent meta-analysis classifies the different presentations of this pathology. ${ }^{14}$ According to it, the most frequent one is isolated myelitis, which is characterized by swelling and enlargement of the involved spinal segment and hyperintensities on T2weighted and fluid-attenuated inversion recovery (FLAIR) sequences with focal nodular enhancement after gadolinium injection. Conversely, encephalic localization can be declined as encephalitis, meningoencephalitis, encephalomyelitis, abscesses, obstructive hydrocephalus or vasculitis. Subsequently, MRI findings are not homogeneous, but, accordingly to the lesion, MRI can show single or multiple subcortical, cortical, or white matter hyperintense lesions on FLAIR and T2WI, usually isointense or hypointense in T1WI, without specific enhancement after gadolinium injection.

While the myelitis due to toxocariasis has been extensively described and reported ${ }^{15,16}$ with MRI findings similar to those quoted above, the encephalic involvement from toxocariasis is currently subject of study. A case report from Spain ${ }^{17}$ described an nonspecific lesion of the cerebellum similar to the one described in our patient A; even in that case, the interpretation was not clear until serodiagnosis confirmed the presence of $T$. canis. Likewise, the MRI findings in our patient were submitted to neurologists and infectious disease experts, but the vasculitic pattern was connected to toxocariasis only after the result of the serodiagnosis.

Regarding the therapy, according to the literature, for patients with moderate to severe symptoms due to VLM, the first-line treatment consists of albendazole $(800 \mathrm{mg} /$ die i.e. $400 \mathrm{mg}$ orally with fatty meal twice daily for five days). ${ }^{18}$ However, despite a large consensus on this therapy, the use of albendazole in the treatment of this disease is still off label in the United States. ${ }^{19}$ Contraindications of the drug include hypersensitivity to the molecule, benzimidazoles, or any component of the formulation. Adverse effects include bone marrow suppression, especially in patients with hepatic impairment and transaminase elevations. Since the conditions of the patients were so severe, they were treated for only 28 days. In the case of OLM patients presenting with sightthreatening ocular inflammation and/or severe multiple-organ involvement, corticosteroids (e.g. prednisone) should be administered before and after initiation of albendazole therapy to minimize inflammatory reactions and prevent increased intracranial pressure. ${ }^{1}$ This therapy was actually started for patient A, while it was avoided for patient $\mathrm{B}$ due to the fact that she had already undergone a long-term steroid treatment with side effects. In this regard, it should also be pointed out that in case of retinal lesions, there would have been the risk of further retinal damage due to albendazole-induced inflammatory changes to the retinal lesions. ${ }^{1}$ However, the severe conditions of both patients made it necessary to start the therapy anyway.

\section{Conclusions}

Both of our patients could not be labeled as a classical presentation of toxocariasis, as there was 
neither eosinophilia nor non-specific lesions of common infection sites such as the brain. Other key challenges in diagnosis were due to the presence of acute comorbidities (e.g. sepsis, pulmonary embolism, epilepsy, vertebral fracture, and recent myocardial infarction), which hampered disease management, prevented clinicians from performing invasive diagnostic procedures, and also withdrew the attention from the main diagnosis.

Given that the presentation of toxocariasis can be so elusive, at least in our experience, clinicians should be made aware of the fact that the occurrence of severe neurological symptoms associated with uveitis and non-specific multisystemic involvement, especially vasculitic or granulomatous reactions, could be suggestive of parasitic infection, even in the absence of eosinophilia. This hypothesis should be particularly considered in Northern Italy and country settings, given the high prevalence of $T$. canis in the dog population.

\section{References}

1. Weller PF, Leder K. Toxocariasis: visceral and ocular larva migrans. Up-to-date Jan 26, 2018. Available from: https://www.uptodate.com/contents/toxocariasis-visceraland-ocular-larva-migrans?source=related_link

2. Pivetti-Pezzi P. Ocular toxocariasis. Int J Med Sci 2009;6:129-30.

3. Paoletti B, Traversa D, Iorio I, et al. Zoonotic parasites in feces and fur of stray and private dogs from Italy. Parasitol Res 2015;114:2135-41.

4. Habluetzel A, Traldi G, Ruggieri S, et al. An estimation of Toxocara canis prevalence in dogs, environmental egg contamination and risk of human infection in the Marche region of Italy. Vet Parasitol 2003;113:243-52.

5. Rubinsky-Elefant G, Hirata CE, Yamamoto JH, Ferreira MU. Human toxocariasis: diagnosis, worldwide seroprevalences and clinical expression of the systemic and ocular forms. Ann Trop Med Parasitol 2010;104:3.

6. Fan CK, Holland CV, Loxton K, Barghouth U. Cerebral toxocariasis: silent progression to neurodegenerative disorders? Clin Microbiol Rev 2015;28:663.

7. Gavignet B, Piarroux R, Aubin F, et al. Cutaneous manifestations of human toxocariasis. J Am Acad Dermatol 2008;59:1031.

8. Glickman LT, Magnaval JF, Domanski LM, et al. Visceral larva migrans in French adults: a new disease syndrome? Am J Epidemiol 1987;125:1019.

9. Taylor MR, Keane CT, O'Connor P, et al. Clinical features of covert toxocariasis. Scand J Infect Dis 1987; 19:693.

10. Finsterer J, Auer H. Neurotoxocarosis. Rev Inst Med Trop S Paulo 2007;49:5.

11. Fillauxab J, Magnavalcd J-F. Laboratory diagnosis of human toxocariasis. Vet Parasitol 2013;193:327-36.

12. Sánchez SS, García HH. Clinical and magnetic resonance imaging findings of neurotoxocariasis (Short Survey). Front Neurol 2018;9:53.

13. Stürchler D, Schubarth $P$, Gualzata $M$, et al. Thiabendazole vs. albendazole in treatment of toxocariasis: a clinical trial. Ann Trop Med Parasitol 1989;83:473.

14. Sanchez S, Garcia HH, Nicoletti A. Clinical and magnetic resonance findings in neurotoxocariasis. Front Neurol 2018 [Epub ahead of print].

15. Lee IH, Kim ST, Oh DK. MRI findings of spinal visceral larva migrans of Toxocara canis. Eur J Radiol 2010;75:236-40.

16. Umehara F, Ookatsu H, Hayashi D, et al. MRI studies of spinal visceral larva migrans syndrome. J Neurol Sci 2006;249:7-12.

17. Moreira-Silva SF, Rodrigues MG, Pimenta JL, et al. Toxocariasis of the central nervous system: with report of two cases. Rev Soc Bras Med Trop 2004;37:2.

18. Albendazole: Drug information Lexicomp ${ }^{\circledR}$. Up-to-date Apr 2, 2016. Available from: https://www.uptodate. com/home [Content available upon registration only].

19. Pawlowski Z. Toxocariasis in humans: clinical expression and treatment dilemma. J Helminthol 2001;75:299. 